\title{
Associations of cerebrovascular metabolism genotypes with neuropsychiatric symptoms and age at onset of Alzheimer's disease dementia
}

\author{
Fabricio F. de Oliveira, ${ }^{1}$ Elizabeth S. Chen, ${ }^{2}$ Marilia C. Smith, ${ }^{2}$ Paulo H. Bertolucci ${ }^{1}$ \\ ${ }^{1}$ Departamento de Neurologia e Neurocirurgia, Escola Paulista de Medicina (EPM), Universidade Federal de São Paulo (UNIFESP), \\ São Paulo, SP, Brazil. ${ }^{2}$ Departamento de Morfologia e Genética, EPM, UNIFESP, São Paulo, SP, Brazil.
}

\begin{abstract}
Objective: To study associations of cerebrovascular metabolism genotypes and haplotypes with age at Alzheimer's disease dementia (AD) onset and with neuropsychiatric symptoms according to each dementia stage.

Methods: Consecutive outpatients with late-onset AD were assessed for age at dementia onset and Neuropsychiatric Inventory scores according to Clinical Dementia Rating scores, apolipoprotein E gene (APOE) haplotypes, angiotensin-converting enzyme gene ( $A C E)$ variants rs1800764 and rs4291, low-density lipoprotein cholesterol receptor gene (LDLR) variants rs11669576 and rs5930, cholesteryl ester transfer protein gene (CETP) variants $1422 \mathrm{~V}$ and TaqIB, and liver $\mathrm{X}$ receptor beta gene (NR1H2) polymorphism rs2695121.

Results: Considering 201 patients, only $A P O E-\varepsilon 4$ carriers had earlier dementia onset in multiple correlations, as well as less apathy, more delusions, and more aberrant motor behavior. Both $A C E$ polymorphisms were associated with less intense frontally mediated behaviors. Regarding LDLR variants, carriers of the A allele of rs11669576 had less anxiety and more aberrant motor behavior, whereas carriers of the A allele of rs5930 had less delusions, less anxiety, more apathy, and more irritability. CETP variants that included $\mathrm{G}$ alleles of I422V and TaqlB were mostly associated with less intense frontally mediated behaviors, while severely impaired carriers of the T allele of rs2695121 had more anxiety and more aberrant motor behavior.

Conclusion: Though only APOE haplotypes affected AD onset, cerebrovascular metabolism genotypes were associated with differences in several neuropsychiatric manifestations of AD.
\end{abstract}

Keywords: Alzheimer disease; dementia; cerebrovascular disorders; genetics; neuropsychiatry

\section{Introduction}

Cerebrovascular risk factors play an important role in the pathogenesis of Alzheimer's disease dementia (AD). When present in midlife, pooled cerebrovascular risk factors synergistically increase $A D$ risk and lower the age at $A D$ onset. ${ }^{1}$ As a result, these patients usually present with a combination of $A D$ and vascular neuropathological profiles. ${ }^{2}$ Conversely, we have previously shown that higher cerebrovascular risk in late life leads to cognitive and functional stabilization or even improvement for patients with $A D$, possibly related to enhanced cerebral perfusion, ${ }^{3}$ though some studies have shown that cerebrovascular risk factors may lead to faster cognitive decline. ${ }^{2}$

The astrocyte-secreted apolipoprotein E is a cholesterolbinding lipoprotein that transports cholesterol through cell membranes ${ }^{4}$ and is involved in cholinergic dysfunction, ${ }^{5}$ atherogenesis, and amyloidogenesis, ${ }^{6}$ besides being a

Correspondence: Fabricio Ferreira de Oliveira, Departamento de Neurologia e Neurocirurgia, Escola Paulista de Medicina, Universidade Federal de São Paulo, Rua Botucatu 740, CEP 04023-900, São Paulo, SP, Brazil.

E-mail: fabricioferreiradeoliveira@ hotmail.com

Submitted Apr 26 2016, accepted Jul 05 2016, Epub Jan 122017. high-affinity ligand for the low-density lipoprotein receptor (LDLR) within the central nervous system. ${ }^{7}$ The apolipoprotein $\mathrm{E}$ gene $(A P O E)$ is a moderately penetrant gene that is neither a prerequisite nor a sufficient agent for development of $A D$, despite the fact that $A P O E-\varepsilon 4$ is the most important genetic risk factor for incidence ${ }^{8}$ and earlier onset of late-onset $A D,{ }^{1}$ while also affecting behavioral performance ${ }^{2}$ and decreasing prospective physical activity in such patients. ${ }^{3} A P O E-\varepsilon 4$ carriers are also more prone to decreased risk of dementia associated with physical activity, ${ }^{9}$ while neuropsychiatric assessment findings may correlate with specific biomarkers. ${ }^{10}$

Several other genetic variants involved in cerebrovascular metabolism have been associated with AD. The two functional variants of the angiotensin-converting enzyme gene $(A C E)$ with the most significant effects for higher activity and boosted serum levels of the angiotensinconverting enzyme (ACE) are the promoter polymorphisms rs1800764 and rs4291, affecting risk and age at onset of the amnestic phenotype of $A D,{ }^{11}$ as well as cognitive decline and incidence of early-onset hypertension. ${ }^{12}$ The LDLR mediates increased astrocytic expression of $A P O E$ induced by amyloid- $\beta,{ }^{4}$ whereas storage and release of cholesterol depend on the expression of the low-density lipoprotein receptor gene $(L D L R)$, which resides within a 
region linked to $A D$ in 19p13.3; rs $11669576^{7}$ and rs $5930^{13}$ are two of the most important genetic variants of the epidermal growth factor precursor homology domain of $L D L R$ to be associated with disrupted cholesterol metabolism and variability in the risk of AD. Cholesterol governs synaptogenesis and myelin biosynthesis, ${ }^{14}$ while the cholesteryl ester transfer protein (CETP) is associated with reverse cholesterol transport (from tissues to the liver) ${ }^{5}$; protective cholesteryl ester transfer protein gene (CETP) variants lead to lower serum CETP levels and healthier lipid profiles, ${ }^{15}$ though not all studies have shown genetically mediated lifetime cognitive effects. ${ }^{16}$ Nevertheless, the A allele of rs708272 (TaqlB) is associated with lower serum CETP activity and lower coronary heart disease risk, ${ }^{17}$ while the $\mathrm{G}$ allele of rs5882 (1422V) has been associated with lower serum CETP levels and greater white matter integrity in young adults, ${ }^{14}$ as well as with preserved cognitive function in longevity ${ }^{18}$ and less medial temporal lobe atrophy in $A P O E-\varepsilon 4$ carriers with $A D .{ }^{19}$ Moreover, the nuclear liver $X$ receptor $\beta$ (LXR- $\beta$ ) isoform is also expressed in the brain, ${ }^{4}$ acting as a regulator of cholesterol homeostasis, controlling amyloidogenesis, and modulating inhibition of angiotensin II, while several variants of the LXR- $\beta$ gene (NR1H2) close to APOE in chromosome 19 have also been linked with variable risk of $A D .^{20}$

We aimed to examine associations of genetic variants of $A P O E, A C E, L D L R, C E T P$, and intron 2 of $N R 1 H 2$ with age at onset of late-onset $A D$ and with neuropsychiatric symptoms, stratified by stage of dementia.

\section{Methods}

\section{Participants}

In this cross-sectional study, consecutive outpatients with late-onset AD according to U.S. National Institute on AgingAlzheimer's Association criteria ${ }^{21}$ were recruited from the Setor de Neurologia do Comportamento, Hospital São Paulo, Universidade Federal de São Paulo (UNIFESP), São Paulo, SP, Brazil, from October 2010 to February 2013. All outpatients with this diagnosis who were followed during the study period were recruited. Late-onset $A D$ was defined as $A D$ with onset of dementia syndrome after age 60 years. ${ }^{1}$ All patients underwent magnetic resonance imaging (MRI) to evaluate either medial parietal or medial, basal, or lateral temporal atrophy or, in cases of claustrophobia or pacemaker use, a computed tomography (CT) scan to exclude vascular lesions. Patients with history of stroke were not included in the study.

A detailed self-report and/or proxy report was conducted for assessment of age at AD onset, years of schooling, gender, and history of antipsychotic and antidepressant therapy. Information on age at dementia onset was obtained through a review of medical records and confirmed after an interview with the caregiver (preferably a family member), who was required to visit the patient frequently. The time of onset of subjective memory complaints or mild cognitive impairment was not taken into account; rather, only the time of dementia onset (when functional decline actually began) was considered. Use of antipsychotics or antidepressants was quantified in patients who had been receiving them for at least 3 months before the evaluation, to ensure that proper clinical response resulting from stability of drug levels in the central nervous system had been achieved, as well as because follow-up assessments at the study facility are usually conducted in 3-month intervals.

\section{Neuropsychiatric assessment}

A thorough neuropsychiatric evaluation was conducted, with particular attention to caregiver-reported Clinical Dementia Rating $(C D R)^{22}$ and the 10 -item version of the Neuropsychiatric Inventory, which does not take into account night-time behavior disturbances or appetite and eating abnormalities. ${ }^{23}$ Effects of body mass index on age at $A D$ onset $^{1}$ and cognitive and functional change ${ }^{3}$ have been previously assessed in our population, and associations of sleep duration and sleep satisfaction with neuropsychiatric symptoms and $A P O E$ haplotypes have been described elsewhere. ${ }^{24}$ Ratings for the final score of each item in the Neuropsychiatric Inventory took into account the frequency and severity of each patient's behaviors, while the total score was the sum of the subscale scores. All functional and behavioral assessments were conducted on weekday mornings, by the same examiner (FFO).

\section{Genotyping procedures}

Blood samples were collected from all patients into tubes containing $0.1 \%$ ethylenediaminetetraacetic acid (EDTA). Genomic DNA was extracted from these samples using a standard salting-out procedure for determination of $A P O E$ haplotypes (polymorphisms rs7412 and rs429358), ACE genotypes and haplotypes (polymorphisms rs1800764 and rs4291), LDLR genotypes and haplotypes (polymorphisms rs11669576 and rs5930), CETP genotypes and haplotypes (polymorphisms rs5882 and rs708272), and genotypes of rs2695121 (intron 2 of the NR1H2 gene). All single nucleotide polymorphisms (SNPs) were assessed by real-time polymerase chain reactions using TaqMan ${ }^{\circledR}$ SNP Genotyping Assays on the Applied Biosystems ${ }^{\circledR} 7500$ Fast RealTime PCR System (Applied Biosystems ${ }^{\circledR}$, Carlsbad, USA). Standard manufacturer protocols were followed. Genotyping procedures were carried out only after clinical data had been collected from all patients, thus ensuring observer blinding to genetic data.

\section{Outcome measures}

Primarily, we sought to determine associations of genetic variants of $A P O E, A C E, L D L R, C E T P$, and intron 2 of the LXR- $\beta$ gene $N R 1 H 2$ with age at AD onset and neuropsychiatric symptoms, stratified by dementia stage. Secondarily, we also investigated combined associations of gender and schooling with age at $A D$ onset, as well as associations of gender, schooling, age at dementia onset, antipsychotic therapy, and antidepressant therapy with each neuropsychiatric symptom according to CDR scores. ${ }^{22}$ Finally, frequencies and scores for all behavioral symptoms were compared according to stage of dementia. 
Table 1 Demographic and clinical profile $(n=201)$

\begin{tabular}{|c|c|c|c|}
\hline Assessed factors & $\mathrm{n}(\%)$ & Mean $\pm S D$ & Range \\
\hline \multicolumn{4}{|l|}{ Gender } \\
\hline Female & $141(70.1)$ & - & - \\
\hline Male & $60(29.9)$ & - & - \\
\hline Age at dementia onset (years) & - & $73.53 \pm 6.3$ & $60.0-88.0$ \\
\hline Schooling (years) & - & $4.22 \pm 3.7$ & $0-15$ \\
\hline Neuropsychiatric Inventory score (0-120 points) & - & $22.11 \pm 16.3$ & $0-87$ \\
\hline \multicolumn{4}{|l|}{ Antipsychotic therapy (mg/day) } \\
\hline Olanzapine & $1(0.5)$ & $10.00 \pm 0.0$ & $10-10$ \\
\hline Quetiapine & $36(17.9)$ & $70.83 \pm 74.5$ & $25-400$ \\
\hline Risperidone & $10(5.0)$ & $1.90 \pm 0.9$ & $1.0-4.0$ \\
\hline \multicolumn{4}{|l|}{ Antidepressant therapy (mg/day)* } \\
\hline Amitriptyline & $1(0.5)$ & $25.00 \pm 0.0$ & $25-25$ \\
\hline Bupropion & $8(4.0)$ & $150.00 \pm 0.0$ & $150-150$ \\
\hline Citalopram & $18(9.0)$ & $23.33 \pm 7.7$ & $20-40$ \\
\hline Clomipramine & $1(0.5)$ & $25.00 \pm 0.0$ & $25-25$ \\
\hline Escitalopram & $3(1.5)$ & $10.00 \pm 0.0$ & $10-10$ \\
\hline Fluoxetine & $11(5.5)$ & $27.27 \pm 10.1$ & $20-40$ \\
\hline Mirtazapine & $2(1.0)$ & $30.00 \pm 0.0$ & $30-30$ \\
\hline Nortriptyline & $1(0.5)$ & $10.00 \pm 0.0$ & $10-10$ \\
\hline Paroxetine & $8(4.0)$ & $27.50 \pm 14.9$ & $20-60$ \\
\hline Sertraline & $25(12.4)$ & $60.00 \pm 20.4$ & $50-100$ \\
\hline Trazodone & $9(4.5)$ & $61.11 \pm 22.0$ & $50-100$ \\
\hline Venlafaxine & $3(1.5)$ & $175.00 \pm 114.6$ & $75-300$ \\
\hline
\end{tabular}

* Overall, 85 patients (42.3\%) used antidepressants during the evaluation, five of whom were on two antidepressants at the same time: one took bupropion $150 \mathrm{mg} /$ day + trazodone $50 \mathrm{mg} /$ day, one took citalopram $20 \mathrm{mg} /$ day + mirtazapine $30 \mathrm{mg} /$ day, and three took sertraline $50 \mathrm{mg} / \mathrm{day}+$ trazodone $50 \mathrm{mg} / \mathrm{day}$.

$\mathrm{SD}=$ standard deviation.

\section{Statistical analysis}

Associations of $A P O E$ haplotypes, $A C E$ genotypes and haplotypes of rs1800764 and rs4291, LDLR genotypes and haplotypes of rs11669576 and rs5930, CETP genotypes and haplotypes of rs5882 and rs708272, and genotypes of rs2695121 with age at AD onset were investigated independently by way of the Mann-Whitney $U$ test and further assessed in combination in a multiple linear regression model that included male gender and schooling as covariates. Multiple linear regression models including a copy of each minor allele, each copy of $A P O E-\varepsilon 4$, male gender, years of schooling, age at dementia onset, antipsychotic therapy, and antidepressant therapy were employed at each dementia stage to investigate associations of these factors with each behavioral symptom and with Neuropsychiatric Inventory total scores. Each haplotype that was represented in at least three patients was associated with each behavioral symptom and with Neuropsychiatric Inventory total scores at each dementia stage by the Mann-Whitney $U$ test. The threshold of significance was set at $p<0.05$.

\section{Ethical considerations}

This study is part of a larger research project (1067/10) approved by the Ethics Committee of Hospital São Paulo, UNIFESP, in August 2010 (CAAE 0540.0.174.000-10). All invited patients and their legal representatives agreed to participate in the study and provided written informed consent before the evaluation.

\section{Results}

At the end of the recruitment period, 217 patients had been included. Of these, $16(7.4 \%)$ were excluded due to missing genetic information, resulting in a final sample of 201 patients.

Table 1 shows demographic and clinical results for the final sample. More than two-thirds of patients were female, 47 patients $(23.4 \%)$ received antipsychotic treatment, and $85(42.3 \%)$ were on at least one antidepressant.

Table 2 reports genotypes and haplotypes for the final sample. Overall, 108 patients $(53.7 \%)$ were $A P O E-\varepsilon 4$ carriers, while $93(46.3 \%)$ were $A P O E-\varepsilon 4$ non-carriers. All assessed genetic variants were in Hardy-Weinberg equilibrium.

Regarding independent haplotypes that could affect age at dementia onset, we found that $A P O E-\varepsilon 4 / \varepsilon 4$ carriers had earlier dementia onset by 4.31 years $(p=0.001)$, rs5882-AA/rs708272-AG carriers had later dementia onset by 1.77 year $(p=0.048)$, rs5882-AG/rs708272-AA carriers had earlier dementia onset by 4.55 years $(p=0.014)$, and rs11669576-GG/rs5930-GG carriers had later dementia onset by 2.14 years $(p=0.021)$. The only isolated genotype that affected age at $A D$ onset was rs5882-AA: carriers had later dementia onset by 1.76 year $(p=0.018)$, but this analysis did not survive multiple correlations. According to Table 3 , the only variable to affect age at $A D$ onset in a multiple linear regression model was the $A P O E$ haplotype: each copy of $A P O E-\varepsilon 4$ led to earlier dementia onset by 1.728 year $(p=0.012)$.

Tables 4, 5, and 6 show the results of multiple linear regression analyses regarding factors that may affect 
Table 2 Genetic analysis findings $(n=201)$

\begin{tabular}{|c|c|c|}
\hline Genotypes and haplotypes & n (\%) & $p$-value* \\
\hline \multicolumn{3}{|l|}{ APOE haplotypes } \\
\hline$\varepsilon 4 / \varepsilon 4$ & $23(11.4)$ & - \\
\hline$\varepsilon 4 / \varepsilon 3$ & $78(38.8)$ & - \\
\hline$\varepsilon 4 / \varepsilon 2$ & $7(3.5)$ & - \\
\hline$\varepsilon 3 / \varepsilon 3$ & $83(41.3)$ & - \\
\hline$\varepsilon 3 / \varepsilon 2$ & $10(5.0)$ & - \\
\hline$\varepsilon 2 / \varepsilon 2$ & $0(0.0)$ & - \\
\hline \multicolumn{3}{|l|}{ rs1800764 genotypes } \\
\hline $\mathrm{CC}$ & $53(26.4)$ & 0.291 \\
\hline CT & 93 (46.3) & \\
\hline TT & $55(27.3)$ & \\
\hline \multicolumn{3}{|l|}{ rs4291 genotypes } \\
\hline AA & $93(46.3)$ & 0.245 \\
\hline AT & $82(40.8)$ & \\
\hline TT & $26(12.9)$ & \\
\hline \multicolumn{3}{|l|}{$A C E$ haplotypes } \\
\hline rs1800764 CC/rs4291 AA & $7(3.5)$ & - \\
\hline rs1800764 CC/rs4291 AT & $20(10.0)$ & - \\
\hline rs1800764 CC/rs4291 TT & $26(12.9)$ & - \\
\hline rs1800764 CT/rs4291 AA & $31(15.4)$ & - \\
\hline rs1800764 CT/rs4291 AT & 62 (30.9) & - \\
\hline rs1800764 CT/rs4291 TT & $0(0.0)$ & - \\
\hline rs1800764 TT/rs4291 AA & $55(27.3)$ & - \\
\hline rs1800764 TT/rs4291 AT & $0(0.0)$ & - \\
\hline rs1800764 TT/rs4291 TT & $0(0.0)$ & - \\
\hline \multicolumn{3}{|l|}{ rs11669576 genotypes (LDLR8) } \\
\hline AA & $2(1.0)$ & 0.425 \\
\hline$A G$ & $27(13.4)$ & \\
\hline GG & $172(85.6)$ & \\
\hline \multicolumn{3}{|l|}{ rs5930 genotypes ( $L D L R 10)$} \\
\hline AA & $23(11.4)$ & 0.593 \\
\hline$A G$ & $95(47.3)$ & \\
\hline GG & $83(41.3)$ & \\
\hline \multicolumn{3}{|l|}{$L D L R$ haplotypes } \\
\hline rs11669576 AA/rs5930 AA & $0(0.0)$ & - \\
\hline rs11669576 AA/rs5930 AG & $0(0.0)$ & - \\
\hline rs11669576 AA/rs5930 GG & $2(1.0)$ & - \\
\hline rs11669576 AG/rs5930 AA & $0(0.0)$ & - \\
\hline rs11669576 AG/rs5930 AG & $10(5.0)$ & - \\
\hline rs11669576 AG/rs5930 GG & $17(8.5)$ & - \\
\hline rs11669576 GG/rs5930 AA & $23(11.4)$ & - \\
\hline rs $11669576 \mathrm{GG} / \mathrm{rs} 5930 \mathrm{AG}$ & 85 (42.3) & - \\
\hline rs11669576 GG/rs5930 GG & $64(31.8)$ & - \\
\hline \multicolumn{3}{|c|}{ rs2695121 genotypes (NR1H2 gene) } \\
\hline CC & $76(37.8)$ & 0.691 \\
\hline CT & 93 (46.3) & \\
\hline TT & $32(15.9)$ & \\
\hline \multicolumn{3}{|l|}{ rs5882 genotypes (CETP gene) } \\
\hline AA & $75(37.3)$ & 0.412 \\
\hline AG & $100(49.8)$ & \\
\hline GG & $26(12.9)$ & \\
\hline \multicolumn{3}{|c|}{ rs708272 genotypes (CETP gene) } \\
\hline AA & $21(10.5)$ & 0.058 \\
\hline$A G$ & $106(52.7)$ & \\
\hline GG & $74(36.8)$ & \\
\hline \multicolumn{3}{|l|}{ CETP haplotypes } \\
\hline rs5882 AA/rs708272 AA & $3(1.5)$ & - \\
\hline rs5882 AA/rs708272 AG & 38 (18.9) & - \\
\hline rs5882 AA/rs708272 GG & $34(16.9)$ & - \\
\hline rs5882 AG/rs708272 AA & $11(5.5)$ & - \\
\hline rs5882 AG/rs708272 AG & $56(27.8)$ & - \\
\hline rs5882 AG/rs708272 GG & $33(16.4)$ & - \\
\hline rs5882 GG/rs708272 AA & $7(3.5)$ & - \\
\hline rs5882 GG/rs708272 AG & $12(6.0)$ & - \\
\hline rs5882 GG/rs708272 GG & $7(3.5)$ & - \\
\hline
\end{tabular}

$A C E=$ angiotensin-converting enzyme gene; $A P O E=$ apolipoprotein $\mathrm{E}$ gene; $C E T P=$ cholesteryl ester transfer protein gene; $L D L R=$ low-density lipoprotein cholesterol receptor gene; $N R 1 H 2$ = liver $\mathrm{X}$ receptor beta gene.

* Hardy-Weinberg equilibrium (chi-square test).
Table 3 Multiple linear regression for age at $A D$ onset $(\mathrm{n}=201)$

\begin{tabular}{lcccc}
\hline & & \multicolumn{2}{c}{$95 \% \mathrm{Cl}$} & \\
\cline { 3 - 4 } Effects & $\beta$ & Lower & Upper & p-value \\
\hline Constant & 75.099 & 72.513 & 77.686 & $<\mathbf{0 . 0 0 1}$ \\
Male gender & -1.473 & -3.419 & 0.472 & 0.137 \\
Years of schooling & 0.134 & -0.113 & 0.381 & 0.285 \\
Copies of $A P O E-\varepsilon 4$ & -1.728 & -3.071 & -0.385 & $\mathbf{0 . 0 1 2}$ \\
Copies of rs1800764-C & 0.608 & -1.119 & 2.335 & 0.488 \\
Copies of rs4291-T & 0.310 & -1.502 & 2.122 & 0.736 \\
Copies of rs11669576-A & -1.177 & -3.562 & 1.207 & 0.331 \\
Copies of rs5930-A & -1.251 & -2.601 & 0.099 & 0.069 \\
Copies of rs2695121-T & 0.745 & -0.516 & 2.006 & 0.246 \\
Copies of rs5882-G & -0.668 & -2.039 & 0.703 & 0.338 \\
Copies of rs708272-A & -0.530 & -1.963 & 0.902 & 0.466 \\
\hline
\end{tabular}

F-ratio $=2.044$; multiple $R=0.312$; adjusted squared multiple $\mathrm{R}=0.050 ; \mathrm{p}=0.031$.

$95 \% \mathrm{Cl}=95 \%$ confidence interval; $\mathrm{AD}=$ Alzheimer's disease dementia; $A P O E$ = apolipoprotein $\mathrm{E}$ gene.

Results in bold are significant.

each behavioral symptom and Neuropsychiatric Inventory total score for patients who were mildly impaired (CDR = $1.0)$, moderately impaired $(C D R=2.0)$, and severely impaired $(C D R=3.0)$, respectively. Overall, Neuropsychiatric Inventory total scores increased according to dementia severity. For mildly impaired patients $(n=78)$, anxiety was the most frequent and highest-scored symptom, while euphoria was the least frequent and hallucinations were the lowest-scored symptom. For moderately impaired patients $(n=92)$, apathy was the most frequent and the highest-scored symptom, while euphoria was the least frequent and hallucinations were the lowest-scored symptom. For severely impaired patients $(n=31)$, apathy was the most frequent and highest-scored symptom, while euphoria was the least frequent and lowest-scored symptom.

Considering associations of independent haplotypes with behavioral scores in mildly impaired patients, rs1800764-CC/rs4291-AT carriers $(n=10)$ had more hallucinations $(1.40 \pm 2.6$ versus $0.15 \pm 0.4, p=0.031)$, rs1800764-CT/rs4291-AA carriers $(n=11)$ had more disinhibition $(1.00 \pm 1.3$ versus $0.39 \pm 1.3, p=0.021)$ and more irritability $(3.00 \pm 4.1$ versus $1.12 \pm 3.0, p=0.022)$, rs $1800764-T$ / rs4291-AA carriers $(n=19)$ had more agitation $(3.32 \pm 3.1$ versus $1.69 \pm 3.1, p=0.017)$, rs11669576-AG/rs5930-AG carriers $(n=5)$ had more apathy $(7.20 \pm 3.6$ versus 3.19 $\pm 3.7, p=0.026), r s 11669576-A G / r s 5930-G G$ carriers $(n=8)$ had less apathy $(1.00 \pm 2.1$ versus $3.73 \pm 3.8$, $\mathrm{p}=0.033)$, rs5882-AA/rs708272-AG carriers $(n=14)$ had lower Neuropsychiatric Inventory total scores (9.86 \pm 6.7 versus $18.66 \pm 11.3, p=0.007)$ and less irritability $(0.57 \pm$ 0.8 versus $2.80 \pm 3.1, p=0.008)$, rs5882-AA/rs708272-GG carriers $(n=13)$ had higher Neuropsychiatric Inventory total scores $(24.38 \pm 9.4$ versus $15.62 \pm 11.0, p=0.007)$ and more apathy $(5.31 \pm 3.4$ versus $3.08 \pm 3.8, p=0.041)$ and more dysphoria (3.38 \pm 3.8 versus $1.34 \pm 2.2, p=0.036)$, rs5882-AG/ rs708272-GG carriers $(n=12)$ had less anxiety $(1.42 \pm 2.7$ versus $4.39 \pm 4.5, p=0.025)$, and rs5882-GG/rs708272-AG carriers $(n=5)$ had more delusions $(3.40 \pm 5.1$ versus $0.58 \pm 1.7, p=0.027)$. 


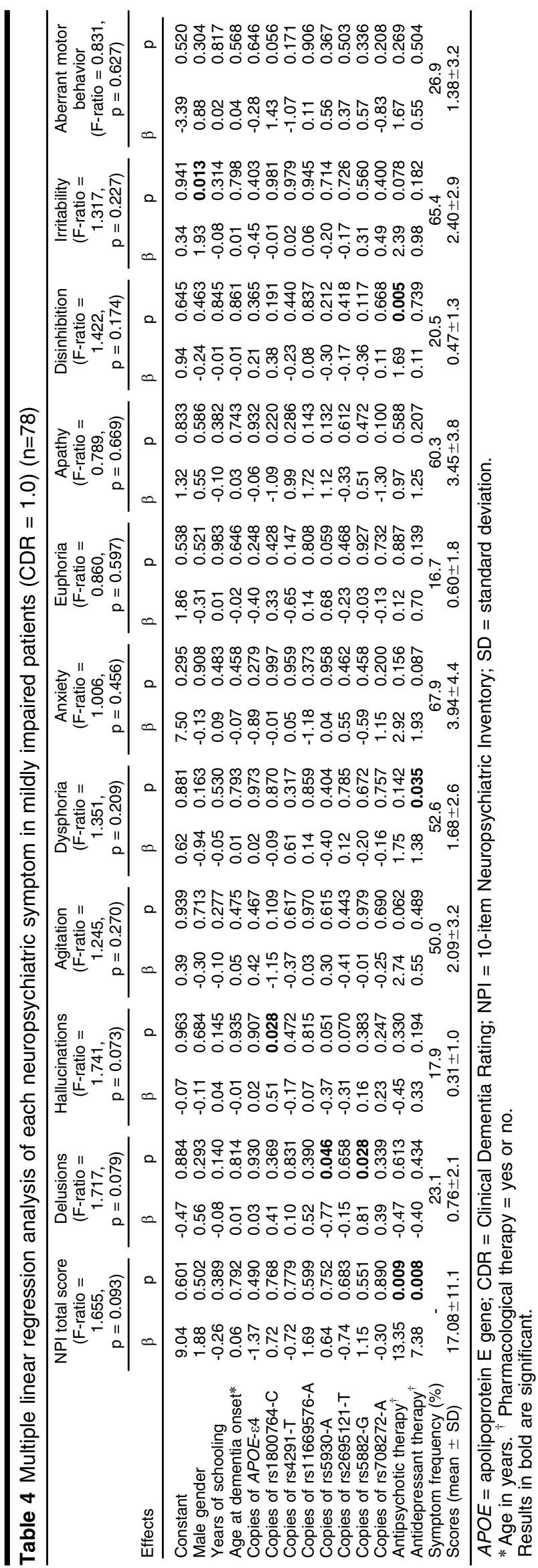

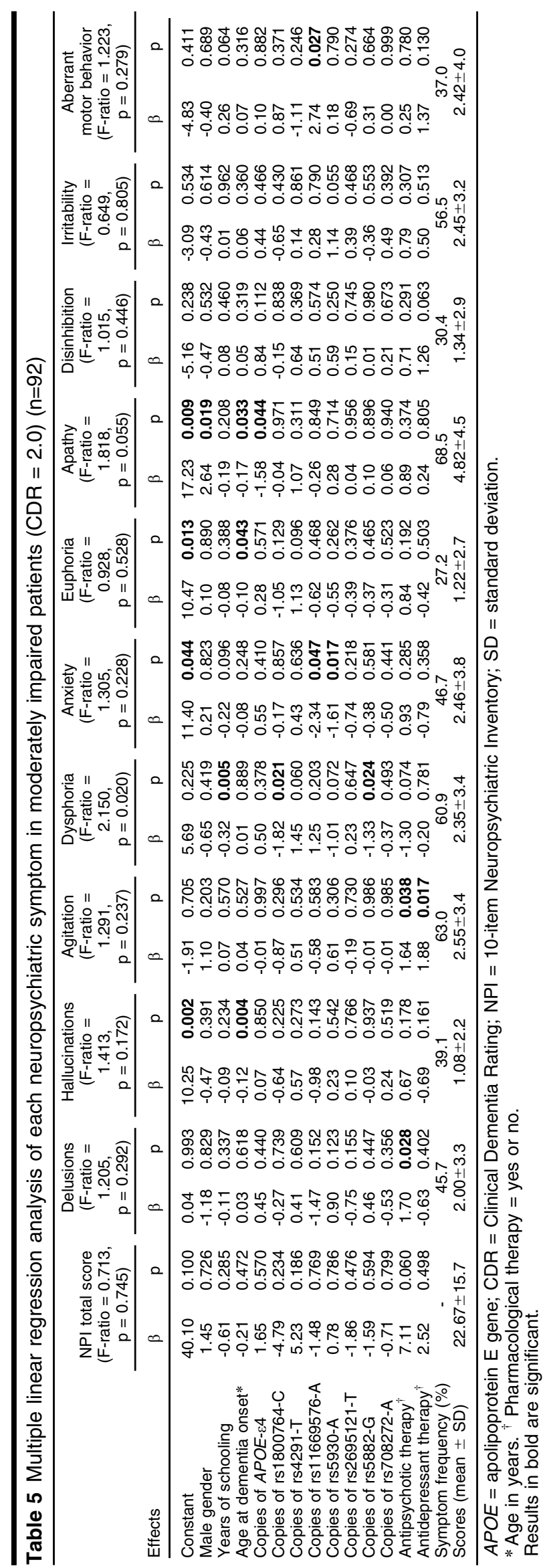


Considering associations of independent haplotypes with behavioral scores in moderately impaired patients, rs 1800764-CC/rs4291-AT carriers $(n=8)$ had less agitation (0.37 \pm 0.7 versus $2.76 \pm 3.5, \mathrm{p}=0.018)$, rs11669576-AG/ rs5930-GG carriers $(n=7)$ had less apathy $(2.00 \pm 4.5$ versus $5.05 \pm 4.5, p=0.049$ ), rs11669576-GG/rs5930-AA carriers $(n=8)$ had less anxiety $(0.12 \pm 0.4$ versus $2.68 \pm$ 3.9, $p=0.033)$, rs11669576-GG/rs5930-AG carriers $(n=42)$ had less irritability $(3.26 \pm 3.7$ versus $1.76 \pm 2.6, p=0.034)$, rs 11669576-GG/rs5930-GG carriers $(n=30)$ had less irritability $(1.37 \pm 2.4$ versus $2.97 \pm 3.4, p=0.006)$, rs5882-AG/ rs708272-AA carriers $(n=8)$ had more agitation $(4.75 \pm 2.9$ versus $2.35 \pm 3.4, \quad p=0.008)$ and more hallucinations $(3.00 \pm 3.8$ versus $0.89 \pm 1.9, p=0.018)$ and more disinhibition ( $4.00 \pm 5.3$ versus $1.08 \pm 2.5, p=0.029)$, and rs5882-AG/ rs708272-AG carriers $(n=23)$ had less agitation (1.39 \pm 2.9 versus $2.94 \pm 3.5, p=0.012$ ).

Considering associations of independent haplotypes with behavioral scores in severely impaired patients, rs1800764-CT/rs4291-AA carriers $(n=3)$ had more aberrant motor behavior (9.33 \pm 2.3 versus $3.14 \pm 4.6, p=0.033)$, rs1800764-CT/rs4291-AT carriers $(n=11)$ had less disinhibition ( $1.27 \pm 2.9$ versus $3.35 \pm 4.0, p=0.038)$, rs $1800764-T \mathrm{~T} /$ rs4291-AA carriers $(n=12)$ had more disinhibition $(4.25 \pm 4.5$ versus $1.58 \pm 2.8, p=0.029$ ), rs5882-AA/rs708272-AG carriers $(n=5)$ had more anxiety $(8.00 \pm 5.7$ versus $2.35 \pm 3.6$, $\mathrm{p}=0.023)$, rs5882-AG/rs708272-AG carriers $(n=11)$ had more irritability $(6.09 \pm 4.1$ versus $2.30 \pm 3.6, p=0.028)$, and rs5882-AG/rs708272-GG carriers $(n=8)$ had less delusions $(0.25 \pm 0.5$ versus $3.00 \pm 3.9, p=0.017)$.

\section{Discussion}

With the exceptions of anxiety and irritability, a trend was found for all other behavioral symptoms to be intensified according to functional decline; likewise, most significant correlations were found in later dementia stages. Anxiety is usually inversely correlated with apathy, ${ }^{25}$ whereas overall neuropsychiatric symptoms tend to correlate better with functional independence than with cognitive decline. ${ }^{25,26}$ Antipsychotic therapy was associated with higher Neuropsychiatric Inventory total scores for mildly impaired patients, particularly regarding disinhibition, and with more delusions and more agitation for moderately impaired patients, probably representing the need for psychotropic treatment when more behavioral symptoms are present, while agitation does not usually improve with atypical antipsychotics in the long term. ${ }^{26}$ Likewise, antidepressant therapy was associated with higher Neuropsychiatric Inventory total scores for mildly impaired and severely impaired patients (particularly regarding dysphoria), and with more agitation for moderately impaired patients, also representing the expected need for such therapy according to symptoms of depression.

Male gender was associated with more irritability in mildly impaired patients, more apathy in moderately impaired patients, and higher Neuropsychiatric Inventory total scores, particularly regarding delusions, in severely impaired patients. Gender differences have been reported in other studies, particularly regarding greater anxiety and dysphoria in women. ${ }^{6}$ 
Schooling did not correlate with dementia onset in this sample. Nevertheless, more years of education were associated with less dysphoria in moderately impaired patients and with lower Neuropsychiatric Inventory total scores in severely impaired patients, particularly regarding agitation and irritability. Though an earlier study showed that educational attainment and AD duration did not affect behavior, ${ }^{25}$ patients were not stratified by dementia stage, and some associations may have been missed. Education enhances cognitive reserve and may affect the way the brain copes with the burden of $A D$ neuropathology, thus modulating behavior.

Later dementia onset was associated with fewer hallucinations, less euphoria, and less apathy for moderately impaired patients. Considering that hallucinations are strong predictors of faster functional decline,${ }^{26}$ they may also be more frequent when dementia starts earlier. A high prevalence of hallucinations and extrapyramidal signs might predict the presence of Lewy bodies in patients with $A D .^{27}$ In severely impaired patients, later dementia onset was associated with higher Neuropsychiatric Inventory total scores, particularly regarding disinhibition, but also with less apathy and less aberrant motor behavior, a complex link that could be partly due to $A P O E-\varepsilon 4$ non-carriers having later $A D$ onset.

On analysis of multiple correlations, only $A P O E$ haplotypes affected the age at dementia onset in this sample, confirming the essential role of genetically mediated neurodegeneration on $A D$ onset. ${ }^{1}$ Furthermore, moderately impaired $A P O E-\varepsilon 4$ carriers had less apathy, whereas severely impaired $A P O E-\varepsilon 4$ carriers had more delusions and more aberrant motor behavior. Whereas some cross-sectional studies have not found associations of $A P O E$ haplotypes with specific neuropsychiatric symptoms ${ }^{25}$ or sleep satisfaction, ${ }^{24}$ other studies have shown dose-dependent associations of copies of $A P O E-\varepsilon 4$ with delusions, ${ }^{28}$ aberrant motor behavior, ${ }^{6}$ anxiety, ${ }^{29}$ and agitation. ${ }^{30}$ Mechanisms involve atrophy of the prefrontal cortex $^{29}$ temporal lobe hypoperfusion, ${ }^{28}$ cholinergic imbalance, ${ }^{6}$ and tau-mediated disruption of frontal serotonergic and dopaminergic networks. ${ }^{30}$ APOE haplotypes have also been associated with variable frequency of hallucinations. ${ }^{6,28}$ The incidence of delusions and hallucinations tends to be dissociated, considering that in $A D$ delusions are usually more common than hallucinations, while in Lewy body dementia syndromes, hallucinations are more common than delusions. ${ }^{31}$ Though $A P O E-\varepsilon 4$ carrier status seems to predispose to late-onset depression, ${ }^{3}$ particularly in women, ${ }^{32}$ we did not find correlations with dysphoria in our sample. Nevertheless, one study showed that women have earlier AD onset when they are $A P O E-\varepsilon 4$ carriers with a history of depression, ${ }^{33}$ confirming that gender differences might affect dementia onset.

Considering ACE genotypes, carriers of the $\mathrm{C}$ allele of rs1800764 had more hallucinations when mildly impaired and less dysphoria when moderately impaired, whereas carriers of the T allele of rs4291 had less dysphoria when severely impaired. Regarding represented haplotypes in independent analyses, presence of the $\mathrm{C}$ allele of rs1800764 was associated with less agitation, while presence of the A allele of rs4291 was associated with more agitation, more disinhibition, more irritability, and worse aberrant motor behavior. To the best of our knowledge, these are all previously unreported associations, while only the T allele of rs4291 was reported as a susceptibility factor for unipolar major depression and hypothalamic-hypophyseal-adrenocortical axis hyperactivity in an earlier study of subjects without dementia. ${ }^{34}$ Given that angiotensin-converting enzyme degrades amyloid- $\beta^{35}$ and is overexpressed in a compensatory manner in the hippocampus, frontal cortex, and caudate nucleus of both hemispheres of patients with $A D,^{12}$ suppression of frontally mediated behaviors could occur by boosted enzyme levels and activity. Functional studies are needed to confirm these associations.

Considering $L D L R$ genotypes, carriers of the A allele of rs11669576 had less anxiety and more aberrant motor behavior when moderately impaired, whereas carriers of the A allele of rs5930 had less delusions when mildly impaired, less anxiety when moderately impaired, and higher Neuropsychiatric Inventory total scores when severely impaired, particularly regarding apathy and irritability. Regarding represented haplotypes in independent analyses, presence of the G allele of rs5930 was associated with less apathy in mildly and moderately impaired patients. Still, carriers of rs11669576-GG/rs5930-GG had later dementia onset. The A allele of rs11669576 has been associated with variable risk of $A D$, either increasing risk when in combination with copies of $A P O E-\varepsilon 4^{7}$ or decreasing risk - only in women - when in combination with other $L D L R$ polymorphisms, ${ }^{36}$ while the $A$ allele of $r 55930$ was associated with lower risk of $A D$ when combined with other $L D L R$ polymorphisms in an earlier study. ${ }^{13}$ Although neuropsychiatric symptoms have not previously been studied in association with variants of $L D L R$, genotypes associated with less $A D$ neuropathology ${ }^{36}$ could lead to later dementia onset and milder behavioral burden in earlier stages.

Considering CETP genotypes, carriers of the G allele of rs5882 had more delusions when mildly impaired, less dysphoria when moderately impaired, and less anxiety when severely impaired, whereas carriers of the A allele of rs708272 had higher Neuropsychiatric Inventory total scores when severely impaired, particularly regarding delusions, anxiety, and aberrant motor behavior. Regarding represented haplotypes in independent analyses, mildly impaired carriers of rs5882-AA/rs708272-GG had higher Neuropsychiatric Inventory total scores, particularly regarding apathy and dysphoria, whereas moderately impaired carriers of rs5882-AG/rs708272-AA had more agitation, more hallucinations, and more disinhibition. Carriers of rs5882-AG/rs708272-AG had less agitation when moderately impaired and more irritability when severely impaired. Carriers of rs5882-AA had later dementia onset, but this finding did not survive multiple correlations; conversely, carriers of rs5882-AG/rs708272AA had earlier dementia onset on independent analysis. Though associations of CETP genotypes with behavioral symptoms have not been reported before, the AA genotype of rs708272 was associated with increased risk of $A D$ for $A P O E-\varepsilon 4$ carriers in one meta-analysis, ${ }^{37}$ while the GG genotype of rs5882 has been associated either with slower cognitive decline and lower risk of $A D,{ }^{15}$ with faster 
cognitive decline and higher risk of $A D,{ }^{38}$ or with increased risk of $A D$ only for $A P O E-\varepsilon 4$ non-carriers. ${ }^{39}$ The $G$ allele of rs708272 and the $G$ allele of rs5882 seemed to play protective roles in our sample, possibly by improving myelin biosynthesis in the brain.

Severely impaired carriers of the T allele of rs2695121 had more anxiety and more aberrant motor behavior. Among all polymorphisms of the LXR- $\beta$ gene (NR1H2), the $T$ allele of rs2695121 is the most significant for risk of $A D,{ }^{20}$ though it had not been previously associated with behavioral symptoms. Polymorphisms in cellular cholesterol efflux-related genes may affect the neuropsychiatric profile of patients with $A D$, as increased cholesterol levels induce amyloidogenesis.

An important limitation of this study is that it was conducted in a single center with no randomization. We tried to mitigate this drawback by ensuring that examiners were blinded to genetic data during the evaluations. In addition, the genotype and haplotype frequencies found were similar to those reported in most previous studies, confirming that our outpatient sample was probably representative of our population as a whole. The cross-sectional design of this study precludes deeper assumptions regarding causal relations. Even though $A P O E$ haplotypes were the only genetic factors to consistently affect age at AD onset, variants involved in cerebrovascular metabolism were associated with several behavioral symptoms, leading to the conclusion that cerebrovascular risk plays an important genetically mediated role in neuropsychiatric manifestations of $A D$.

\section{Acknowledgements}

This work was sponsored by Coordenação de Aperfeiçoamento de Pessoal de Nível Superior (CAPES) (grant 1067/10) and Fundação de Amparo à Pesquisa do Estado de São Paulo (FAPESP) (grant 2015/10109-5). FFO has received research support from CAPES and FAPESP. MCS has received grants from CAPES, Conselho Nacional de Desenvolvimento Científico e Tecnológico (CNPq), and FAPESP. PHB has received grants from FAPESP.

\section{Disclosure}

FFO serves as a healthcare council member for Gerson Lehrman Group. PHB has served as a consultant for Lundbeck, Novartis, and Support. The other authors report no conflicts of interest.

\section{References}

1 de Oliveira FF, Bertolucci PH, Chen ES, Smith MC. Risk factors for age at onset of dementia due to Alzheimer's disease in a sample of patients with low mean schooling from São Paulo, Brazil. Int J Geriatr Psychiatry. 2014;29:1033-9.

2 Ferreira LK, Tamashiro-Duran JH, Squarzoni P, Duran FL, Alves TC, Buchpiguel CA, et al. The link between cardiovascular risk, Alzheimer's disease, and mild cognitive impairment: support from recent functional neuroimaging studies. Rev Bras Psiquiatr. 2014;36:344-57.

3 de Oliveira FF, Pivi GA, Chen ES, Smith MC, Bertolucci PH. Risk factors for cognitive and functional change in one year in patients with Alzheimer's disease dementia from São Paulo, Brazil. J Neurol Sci. 2015;359:127-32.
4 Carter CJ. Convergence of genes implicated in Alzheimer's disease on the cerebral cholesterol shuttle: APP, cholesterol, lipoproteins, and atherosclerosis. Neurochem Int. 2007;50:12-38.

5 Martins IJ, Berger T, Sharman MJ, Verdile G, Fuller SJ, Martins RN. Cholesterol metabolism and transport in the pathogenesis of Alzheimer's disease. J Neurochem. 2009;111:1275-308.

6 Del Prete M, Spaccavento S, Craca A, Fiore P, Angelelli P. Neuropsychiatric symptoms and the APOE genotype in Alzheimer's disease. Neurol Sci. 2009;30:367-73.

7 Retz W, Thome J, Durany N, Harsányi A, Retz-Junginger $P$, Kornhuber J, et al. Potential genetic markers of sporadic Alzheimer's dementia. Psychiatr Genet. 2001;11:115-22.

8 Bilbul M, Schipper HM. Risk Profiles of Alzheimer disease. Can J Neurol Sci. 2011;38:580-92.

9 Rovio S, Kåreholt I, Helkala EL, Viitanen M, Winblad B, Tuomilehto J, et al. Leisure-time physical activity at midlife and the risk of dementia and Alzheimer's disease. Lancet Neurol. 2005;4:705-11.

10 Braun M, Tupper D, Kaufmann P, McCrea M, Postal K, Westerveld $M$, et al. Neuropsychological assessment: a valuable tool in the diagnosis and management of neurological, neurodevelopmental, medical, and psychiatric disorders. Cogn Behav Neurol. 2011;24: 107-14.

11 Vardy ER, Brown K, Stopford CL, Thompson JC, Richardson AM, Neary D, et al. Cognitive phenotypes in Alzheimer's disease and genetic variants in ACE and IDE. Neurobiol Aging. 2012;33:1486.e1-2.

12 de Oliveira FF, Bertolucci PH, Chen ES, Smith MC. Brain-penetrating angiotensin-converting enzyme inhibitors and cognitive change in patients with dementia due to Alzheimer's disease. J Alzheimers Dis. 2014;42:S321-4.

13 Gopalraj RK, Zhu H, Kelly JF, Mendiondo M, Pulliam JF, Bennett DA, et al. Genetic association of low density lipoprotein receptor and Alzheimer's disease. Neurobiol Aging. 2005;26:1-7.

14 Warstadt NM, Dennis EL, Jahanshad N, Kohannim O, Nir TM, McMahon KL, et al. Serum cholesterol and variant in cholesterolrelated gene CETP predict white matter microstructure. Neurobiol Aging. 2014;35:2504-13.

15 Sanders AE, Wang C, Katz M, Derby CA, Barzilai N, Ozelius L, et al. Association of a functional polymorphism in the cholesteryl ester transfer protein (CETP) gene with memory decline and incidence of dementia. JAMA. 2010;303:150-8.

16 Johnson W, Harris SE, Collins P, Starr JM, Whalley LJ, Deary IJ. No association of CETP genotype with cognitive function or age-related cognitive change. Neurosci Lett. 2007;420:189-92.

17 Thompson A, Di Angelantonio E, Sarwar N, Erqou S, Saleheen D, Dullaart RP, et al. Association of cholesteryl ester transfer protein genotypes with CETP mass and activity, lipid levels, and coronary risk. JAMA. 2008;299:2777-88.

18 Barzilai N, Atzmon G, Derby CA, Bauman JM, Lipton RB. A genotype of exceptional longevity is associated with preservation of cognitive function. Neurology. 2006;67:2170-5.

19 Murphy EA, Roddey JC, McEvoy LK, Holland D, Hagler DJ Jr, Dale $A M$, et al. CETP polymorphisms associate with brain structure, atrophy rate, and Alzheimer's disease risk in an APOE-dependent manner. Brain Imaging Behav. 2012;6:16-26.

20 Infante J, Rodríguez-Rodríguez E, Mateo I, Llorca J, Vázquez-Iguera $\mathrm{JL}$, Berciano J, et al. Gene-gene interaction between heme oxygenase- 1 and liver $X$ receptor- $\beta$ and Alzheimer's disease risk. Neurobiol Aging. 2010;31:710-4.

21 McKhann GM, Knopman DS, Chertkow H, Hyman BT, Jack CR Jr, Kawas $\mathrm{CH}$, et al. The diagnosis of dementia due to Alzheimer's disease: recommendations from the National Institute on AgingAlzheimer's Association workgroups on diagnostic guidelines for Alzheimer's disease. Alzheimers Dement. 2011;7:263-9.

22 Chaves ML, Camozzato AL, Godinho C, Kochhann R, Schuh A, de Almeida VL, et al. Validity of the clinical dementia rating scale for the detection and staging of dementia in Brazilian patients. Alzheimer Dis Assoc Disord. 2007;21:210-7.

23 Camozzatto AL, Kochhann R, Simeoni C, Konrath CA, Pedro Franz A, Carvalho A, et al. Reliability of the Brazilian Portuguese version of the Neuropsychiatric Inventory (NPI) for patients with Alzheimer's disease and their caregivers. Int Psychogeriatr. 2008;20:383-93.

24 de Oliveira FF, Bertolucci PH, Chen ES, Smith Mde A. Assessment of sleep satisfaction in patients with dementia due to Alzheimer's disease. J Clin Neurosci. 2014;21:2112-7. 
25 de Oliveira FF, Wajman JR, Bertolucci PH, Chen ES, Smith MC. Correlations among cognitive and behavioural assessments in patients with dementia due to Alzheimer's disease. Clin Neurol Neurosurg. 2015;135:27-33.

26 de Oliveira FF, Bertolucci PH, Chen ES, Smith Mde A. Pharmacological modulation of cognitive and behavioral symptoms in patients with dementia due to Alzheimer's disease. J Neurol Sci. 2014;336:103-8.

27 lqbal K, Flory M, Soininen $\mathrm{H}$. Clinical symptoms and symptom signatures of Alzheimer's disease subgroups. J Alzheimers Dis. 2013; 37:475-81.

28 Scarmeas N, Brandt J, Albert M, Devanand DP, Marder K, Bell K, et al. Association between the APOE genotype and psychopathologic symptoms in Alzheimer's disease. Neurology. 2002;58:1182-8.

29 Yoo HB, Lee HW, Shin S, Park SW, Choi JS, Jung HY, et al. Butyrylcholinesterase $\mathrm{K}$ and apolipoprotein $\varepsilon 4$ affect cortical thickness and neuropsychiatric symptoms in Alzheimer's disease. Curr Alzheimer Res. 2014;11:137-44.

30 Craig D, Hart DJ, McKool K, Mcllroy SP, Passmore AP. Apolipoprotein E e4 allele influences aggressive behaviour in Alzheimer's disease. J Neurol Neurosurg Psychiatry. 2004;75:1327-30.

31 Oliveira FF, Machado FC, Sampaio G, Marin SM, Chen ES, Smith $\mathrm{MC}$, et al. Contrasts between patients with Lewy body dementia syndromes and APOE- $83 / \varepsilon 3$ patients with late-onset Alzheimer disease dementia. Neurologist. 2015;20:35-41.

32 Delano-Wood L, Houston WS, Emond JA, Marchant NL, Salmon DP, Jeste DV, et al. APOE genotype predicts depression in women with
Alzheimer's disease: a retrospective study. Int J Geriatr Psychiatry. 2008;23:632-6.

33 de Oliveira FF, Bertolucci PH, Chen ES, Smith MC. Assessment of risk factors for earlier onset of sporadic Alzheimer's disease dementia. Neurol India; 2014;62:625-30.

34 Baghai TC, Binder EB, Schule C, Salyakina D, Eser D, Lucae S, et al. Polymorphisms in the angiotensin-converting enzyme gene are associated with unipolar depression, ACE activity and hypercortisolism. Mol Psychiatry. 2006;11:1003-15.

35 Ning M, Yang Y, Zhang Z, Chen Z, Zhao T, Zhang D, et al. Amyloid$\beta$-related genes SORL1 and ACE are genetically associated with risk for late-onset Alzheimer disease in the Chinese population. Alzheimer Dis Assoc Disord. 2010;24:390-6.

36 Lämsä R, Helisalmi S, Herukka SK, Tapiola T, Pirttilä T, Vepsäläinen $S$, et al. Genetic study evaluating LDLR polymorphisms and Alzheimer's disease. Neurobiol Aging. 2008;29:848-55.

37 Li Q, Huang P, He QC, Lin QZ, Wu J, Yin RX. Association between the CETP polymorphisms and the risk of Alzheimer's disease, carotid atherosclerosis, longevity, and the efficacy of statin therapy. Neurobiol Aging. 2014;35:1513.e13-23.

38 Yu L, Shulman JM, Chibnik L, Leurgans S, Schneider JA, De Jager $\mathrm{PL}$, et al. The CETP I405V polymorphism is associated with an increased risk of Alzheimer's disease. Aging Cell. 2012;11:228-33.

39 Arias-Vásquez A, Isaacs A, Aulchenko YS, Hofman A, Oostra BA, Breteler M, et al. The cholesteryl ester transfer protein (CETP) gene and the risk of Alzheimer's disease. Neurogenetics. 2007;8:189-93. 